\title{
ON THE THEORY OF PLANE STRESS*
}

\author{
$\mathrm{BY}$ \\ EDWARD L. REISS AND STANLEY LOCKE** \\ Institute of Mathematical Sciences, New York University, and Republic Aviation
}

1. Introduction. The classical theory of plane stress [1] is concerned with a thin elastic plate subjected to edge forces which deform it so that there is no normal displacement of its middle plane. This theory is conventionally obtained from the exact three dimensional linear theory of elasticity for homogeneous and isotropic materials, ${ }^{1}$ by neglecting some of the compatibility equations and assuming that the shear and normal stresses, transverse to the middle plane of the plate, vanish and the remaining stresses are independent of $z$, the coordinate normal to this mid-plane. These assumptions imply that only two boundary conditions, which are functions of one variable, are required along the edge. This is in contrast to the three of the exact theory which are functions of two variables. Therefore, a solution of the plane stress theory is not, in general, a solution of the exact theory. ${ }^{2}$ The plane stress solutions can provide accurate approximations if the plate thickness is "small" compared to each of its lateral dimensions.

In this paper, we indicate the relationship between the two theories ${ }^{3}$ by systematically deriving from the exact theory the differential equations and boundary conditions of plane stress. Our technique of derivation also provides a systematic method of obtaining increasingly accurate approximations to the exact stress distribution. This technique was previously developed and applied to other related elasticity problems $[3,4,5]$. In a sense, it is a generalization of the boundary layer method used by Friedrichs [6] and later by Friedrichs and Dressler [7] in a study of the bending of plates. In the latter reference, it is also shown that by decomposing each stress component and edge force into the sum of an even and an odd function of $z$, the exact theory for the general problem of the loaded plate separates into two distinct systems of differential equations and boundary conditions. One system describes the "bending" of plates and is partially treated in [3], [6] and [7]. The second system, which is concerned with the "extension" of plates, is considered in this paper. Hereafter, each stress component and edge force is assumed to possess its specific even or odd property. These are listed at the end of Sec. 2.

Briefly, our method is to expand each stress component in a power series in the plate thickness, $h$. Substituting these expansions into the exact theory and equating coefficients of like powers of $h$ yields a sequence of systems of differential equations to determine the expansion coefficients. The equations of the lowest order approximation (the "zeroth order interior problem") coincide with those of the plane stress theory. Higher order systems provide "corrections" to the plane stress approximation. In

${ }^{*}$ Received November 21,1960 . The work reported in this paper was performed at the Institute of Mathematical Sciences under Contract AT(30-1)-1480 with the United States Atomic Energy Commission and Contract AF-49(638)-161 with the United States Air Force. Reproduction in whole or in part is permitted for any purpose of the United States Government.

**Now at Schlumberger Well Surveying Corporation.

${ }^{1}$ Hereafter referred to as the exact theory.

${ }^{2}$ There are special cases for which the plane stress solution is a solution of the exact theory, e.g. [2].

${ }^{3}$ In this connection see [9] and the discussion in [1]. 
general, the expansions cannot satisfy the boundary conditions of the exact theory along the edge, nor do they represent the stresses in a region adjacent to the edge. ${ }^{4}$ To determine, from the exact theory, appropriate boundary conditions for the previously mentioned differential equations and to obtain approximations for the stresses near the edge, we employ a second expansion in $h$, whose coefficients are now functions of new independent variables. These new variables are obtained by "stretching" the coordinate normal to the edge of the plate. ${ }^{5}$ Substituting these expansions into the exact theory and equating coefficients of like powers of $h$ results in a sequence of boundary value problems, whose solutions yield approximations to the stress distribution near the edge. The requirement of existence of single valued solutions of these problems provides the desired boundary conditions. The boundary conditions obtained from the lowest order approximation coincide with those of the plane stress theory.

In Sec. 6, the procedure for obtaining the higher order approximations for the threedimensional stress distribution is summarized.

2. Formulation. An elastic plate of thickness $2 \mathrm{~h}$ is considered as a three-dimensional elastic body bounded by the planes $z= \pm h$ and the cylindrical surface $x=X(s)$, $y=Y(s)$. The bounding planes $z=h$ and $z=-h$ are referred to as the faces of the plate. The surface, $|z| \leq h, x=X(s), y=Y(s)$, is called the edge, and the "smooth" curve $B: z=0, x=X(s), y=Y(s)$, is called the boundary curve.

For simplicity, it is assumed that the faces are force free ${ }^{6}$ and that the edge is subjected to an arbitrary equilibrium distribution of normal and shear forces. We further assume that the magnitudes of the edge forces are sufficiently small to insure that the exact theory remains valid.

If we introduce the dimensionless variable

$$
\zeta=\frac{z}{h}
$$

then the faces are given by $\zeta= \pm 1$. If the stresses are functions of $x, y$ and $\zeta$ the equations of the exact theory are [1]

Equilibrium equations,

$$
\begin{aligned}
& \sigma_{z x, \zeta}+h\left[\sigma_{x, x}+\sigma_{x y, y}\right]=0, \quad \sigma_{z y, \zeta}+h\left[\sigma_{x y, x}+\sigma_{y, y}\right]=0, \\
& \sigma_{z, \zeta}+h\left[\sigma_{z x, x}+\sigma_{z y, y}\right]=0 ;
\end{aligned}
$$

Compatibility equations,

$$
\begin{array}{ll}
\sigma_{x, \zeta 5}+h^{2}\left[\Delta \sigma_{x}+\Omega_{, x x}\right]=0, & \sigma_{y, \zeta 5}+h^{2}\left[\Delta \sigma_{y}+\Omega_{, y y}\right]=0, \\
\sigma_{z, \zeta 5}+\Omega_{. \zeta 5}+h^{2} \Delta \sigma_{z}=0, & \sigma_{z x, \zeta 5}+h \Omega_{. \zeta x}+h^{2} \Delta \sigma_{z x}=0, \\
\sigma_{z y, \zeta 5}+h \Omega_{, \zeta y}+h^{2} \Delta \sigma_{z y}=0, & \sigma_{x y, \zeta 5}+h^{2}\left[\Delta \sigma_{x y}+\Omega_{, x y}\right]=0 .
\end{array}
$$

Here we have employed the notation,

$$
\Delta \equiv \frac{\partial^{2}}{\partial x^{2}}+\frac{\partial^{2}}{\partial y^{2}}, \quad \Omega=\frac{1}{1+\nu}\left(\sigma_{x}+\sigma_{\nu}+\sigma_{z}\right),
$$

${ }^{4}$ See Secs. 3 and 4.

${ }^{5}$ See Sec. 4.

${ }^{6}$ This restriction is not essential, but merely condenses the calculations. 
and an independent variable appearing as a subscript and following a comma indicates partial differentiation with respect to that variable, e.g., $\sigma_{z x, 5}=\partial \sigma_{z x} / \partial \zeta$.

To complete the formulation we require appropriate boundary conditions. These are obtained by specifying the applied forces as

$$
\begin{aligned}
& \sigma_{z}(x, y, \pm 1 ; h)=\sigma_{z x}(x, y, \pm 1 ; h)=\sigma_{z y}(x, y, \pm 1 ; h)=0 ; \\
& \sigma_{n}(X, Y, \zeta ; h)=L(s, \zeta ; h), \quad \sigma_{n s}(X, Y, \zeta ; h)=Q(s, \zeta ; h), \\
& \sigma_{n z}(X, Y, \zeta ; h)=R(s, \zeta ; h) .
\end{aligned}
$$

In (4b), $n$ signifies the outward normal to $B$ and $s$ the distance along $B . L, Q$ and $R$ are the prescribed normal and shear edge loads. Since Eqs. (2-4) are the stress formulation of the elastic problem for the plate, no explicit reference to Hooke's law is required.

In this paper attention is focused on the "extension" of the plate by the edge forces. This implies [7] that $L, Q, \sigma_{x}, \sigma_{y}, \sigma_{z}$, and $\sigma_{x y}$ are even functions of $\zeta$, while $R, \sigma_{z x}$ and $\sigma_{z y}$ are odd functions of $\zeta$. In the ensuing analysis we shall make frequent use of the even or odd property of each of these quantities without explicit reference.

3. The interior problems. We assume that each stress component symbolized by, $\sigma(x, y, \zeta ; h)$, can be represented, in an asymptotic sense, by a power series in $h$

$$
\sigma(x, y, \zeta ; h) \sim \sum_{i=0}^{\infty} \sigma^{i}(x, y, \zeta) h^{i} .
$$

Furthermore we define $\sigma^{i}=0$ if $i<0$. The functions $\sigma^{i}(x, y, \zeta)$ are called the interior stress coefficients and are assumed to possess the same even or odd property as $\sigma(x, y, \zeta ; h)$. We also assume that the prescribed edge forces in (4) can be expanded in power series in $h$ :

$$
\left\{\begin{array}{l}
L(s, \zeta ; h) \\
Q(s, \zeta ; h) \\
R(s, \zeta ; h)
\end{array}\right\}=\sum_{i=0}^{\infty}\left\{\begin{array}{l}
L^{i}(s, \zeta) \\
Q^{i}(s, \zeta) \\
R^{i}(s, \zeta)
\end{array}\right\} h^{i} .
$$

Substituting Eqs. (5) into the differential equations (2) and (3) and boundary conditions (4a) and equating coefficients of the same powers of $h$ yields, from the coefficients of $h^{n}$,

$$
\begin{gathered}
\sigma_{z x, \zeta}^{n}+\sigma_{x, x}^{n-1}+\sigma_{x y, y}^{n-1}=0, \quad \sigma_{z y, \zeta}^{n}+\sigma_{x y, x}^{n-1}+\sigma_{y, y}^{n-1}=0, \\
\sigma_{z, \zeta}^{n}+\sigma_{z x, x}^{n-1}+\sigma_{z y, y}^{n-1}=0 ; \\
\sigma_{x, \zeta 5}^{n}+\Delta \sigma_{x}^{n-2}+\Omega_{, x x}^{n-2}=0, \quad \sigma_{y, \zeta 5}^{n}+\Delta \sigma_{y}^{n-2}+\Omega_{, y y}^{n-2}=0, \\
\sigma_{z, \zeta 5}^{n}+\Omega_{, \zeta 5}^{n}+\Delta \sigma_{z}^{n-2}=0, \\
\sigma_{z x, \zeta 5}^{n}+\Omega_{, x \zeta}^{n-1}+\Delta \sigma_{z x}^{n-2}=0, \quad \sigma_{z y, \zeta 5}^{n}+\Omega_{, y \zeta}^{n-1}+\Delta \sigma_{z y}^{n-2}=0 \\
\sigma_{x y, \zeta 5}^{n}+\Delta \sigma_{x y}^{n-2}+\Omega_{, x y}^{n-2}=0 ; \\
\sigma_{z x}^{n}(x, y, \pm 1)=\sigma_{z y}^{n}(x, y, \pm 1)=\sigma_{z}^{n}(x, y, \pm 1)=0 .
\end{gathered}
$$

We now proceed to systematically analyze (7-9). Since $\sigma^{i}=0$ if $i<0$, it follows from (7) and (9) that,

$$
\sigma_{z x}^{0}=\sigma_{z y}^{0}=\sigma_{z}^{0}=\sigma_{z}^{1} \equiv 0
$$


An immediate consequence of $(8 a, b, f)$ and (10) is that,

$$
\begin{gathered}
\sigma_{x}^{n}=S_{x}^{n}(x, y), \quad \sigma_{\nu}^{n}=S_{y}^{n}(x, y), \quad \sigma_{x y}^{n}=S_{x y}^{n}(x, y), \\
(1+\nu) \Omega^{n}(x, y)=S_{x}^{n}(x, y)+S_{y}^{n}(x, y), \quad \text { if } \quad n=0,1 .
\end{gathered}
$$

Equations (7a, b), (9) and (11) then imply that,

$$
\sigma_{z x}^{1}=\sigma_{z y}^{1}=0 .
$$

We can, in fact, prove $^{7}$ that,

$$
\sigma_{z x}^{n}=\sigma_{z y}^{n}=\sigma_{z}^{n}=0, \quad \Omega^{n}=\Omega^{n}(x, y)=\frac{1}{1+\nu}\left(\sigma_{x}^{n}+\sigma_{y}^{n}\right), \quad n=0,1, \cdots .
$$

Hence (7c) and (8c, d, e) are identically satisfied by (13) for all $n$ and (7a, b) become,

$$
\sigma_{x, x}^{n}+\sigma_{x y, y}^{n}=\sigma_{x y, x}^{n}+\sigma_{y, y}^{n}=0, \quad \mathrm{n}=0,1, \cdots .
$$

Furthermore if $\sigma_{x}^{n}$ and $\sigma_{y}^{n}$ satisfy $(8 \mathrm{a}, \mathrm{b})$ then using $(13)$, we see that,

$$
\Delta \Omega^{n}=0, \quad n=0,1, \cdots .
$$

Integration of $(8 \mathrm{a}, \mathrm{b}, \mathrm{f})$ with respect to $\zeta$ yields

$$
\begin{aligned}
& \sigma_{x}^{n}(x, y, \zeta)=S_{x}^{n}(x, y)-\frac{\zeta^{2}}{2} \Omega_{, x x}^{n-2}-\int^{\zeta} \int^{\zeta^{\prime}} \Delta \sigma_{x}^{n-2}(x, y, \beta) d \beta d \zeta^{\prime} \\
& \sigma_{y}^{n}(x, y, \zeta)=S_{y}^{n}(x, y)-\frac{\zeta^{2}}{2} \Omega_{, y y}^{n-2}-\int^{\zeta} \int^{\zeta^{\prime}} \Delta \sigma_{y}^{n-2}(x, y, \beta) d \beta d \zeta^{\prime}, \\
& \sigma_{x y}^{n}(x, y, \zeta)=S_{x y}^{n}(x, y)+\frac{\nu}{2} \zeta^{2} \Omega_{, x y}^{n-2}, \quad n=0,1, \cdots,
\end{aligned}
$$

and (13) as solutions of (7-9) provided that $S_{x}^{n}, S_{y}^{n}$ and $S_{x y}^{n}$ satisfy the following differential equations obtained from (14):

$$
\begin{aligned}
S_{x, x}^{n}+S_{x y, y}^{n} & =\frac{\zeta^{2}}{2} \frac{\partial}{\partial x}\left[\Omega_{, x x}^{n-2}-\nu \Omega_{, y y}^{n-2}\right]+\int^{\zeta} \int^{\zeta^{\prime}} \Delta \sigma_{x, x}^{n-2} d \beta d \zeta^{\prime}, \\
S_{x y, x}^{n}+S_{y, y}^{n} & =\frac{\zeta^{2}}{2} \frac{\partial}{\partial y}\left[\Omega_{, y y}^{n-2}-\nu \Omega_{, x x}^{n-2}\right]+\int^{\zeta} \int^{\zeta^{\prime}} \Delta \sigma_{y, y}^{n-2} d \beta d \zeta^{\prime}, \\
\Delta \Omega^{n} & =\Delta\left(S_{x}^{n}+S_{y}^{n}\right)=0, \quad n=0,1, \cdots .
\end{aligned}
$$

In particular, we observe that (15) reduces to (11) for $n=0,1$ and (16) reduces to

$$
S_{x, x}^{n}+S_{x y, v}^{n}=S_{x y, x}^{n}+S_{y, y}^{n}=\Delta\left(S_{x}^{n}+S_{y}^{n}\right)=0, \text { if } n=0,1 .
$$

Equations (10), (11) and (17) give the equations of the classical theory of plane stress [1]. It is convenient to refer to (17) with $n=0$ and $n=1$ as the differential equations of the zeroth and first order interior problems respectively. By recursive application of (15) and (16) we obtain all higher order interior problems. For example, if $n=2,3$

$$
\begin{aligned}
\sigma_{x}^{n} & =S_{x}^{n}(x, y)-\frac{1}{2}\left[\Delta S_{x}^{n-2}+\Omega_{, x x}^{n-2}\right] \zeta^{2}, \\
\sigma_{y}^{n} & =S_{y}^{n}(x, y)-\frac{1}{2}\left[\Delta S_{y}^{n-2}+\Omega_{, y y}^{n-2}\right] \zeta^{2}, \\
\sigma_{x y}^{n} & =S_{x y}^{n}(x, y)+\frac{\nu}{2} \Omega_{. x y}^{n-2} \zeta^{2}
\end{aligned}
$$

${ }^{7}$ See Appendix. 


$$
S_{x, x}^{n}+S_{x y, y}^{n}=S_{x y, x}^{n}+S_{y, y}^{n}=\Delta\left(S_{x}^{n}+S_{y}^{n}\right)=0 .
$$

4. Formulation of the boundary layer problem. In the previous section we obtained, for all $n$, explicit differential equations, (16), and expressions for the interior stress coefficients (13) and (15). These results were derived from the exact theory independent of the boundary conditions along the edge, $(4 \mathrm{~b})$. For each $n$, the differential equations form a "fourth" order system and hence require two boundary conditions on $B$, which are functions of only $s$. The exact theory requires three conditions, (4b), that are functions of $s$ and $\zeta$. Furthermore we observe that the interior stress coefficients have a specific dependence on $\zeta,(13)$ and (15), while the prescribed stresses in (4b) are arbitrary functions. Therefore the interior expansions (5) cannot, in general, satisfy the edge boundary conditions. In fact, if the series (5) represent the solution they do so in some region away from the edge, i.e. in the "interior" of the plate. Near the edge, i.e. in the "boundary layer," the solutions vary rapidly from those of the interior solution to satisfy the boundary conditions of the exact theory. To obtain an expansion that uniformly represents the solution up to and including the edge we employ a "stretching" of the independent variables. Our procedure, which has previously been employed in studying other related problems [3, 4, 5], differs somewhat from that of Friedrichs [6, 7]. We assume that the rapid variations that occur in the boundary layer depend only on the direction normal to the edge. Therefore, only the independent variable in this direction is stretched. ${ }^{8}$

To facilitate this, consider any point $P$ on $B$ and assume, without loss of generality, that the $x$ and $y$ axes coincide, respectively, with the normal and tangent to $B$ at $P$. We define at $P$ the boundary layer coordinates $\xi, y, \zeta$ by "stretching" the variable $x$ so that,

$$
\xi=\frac{x}{h} .
$$

Then for sufficiently small $h$, any fixed neighborhood of the edge, no matter how small, in the $x$ variable corresponds to an arbitrarily large one in the $\xi$ variable. Roughly this implies that the boundary layer effects penetrate from the edge into the plate a "distance" of order of magnitude $h$.

If $f(\xi, y, \zeta ; h)=\sigma(x, y, \zeta ; h)$ is the generic symbol for the stresses as functions of $\xi, y, \zeta$, we assume that

$$
f(\xi, y, \zeta ; h) \sim \sum_{i=0}^{\infty} f^{i}(\xi, y, \zeta) h^{i}
$$

Here $f^{i}$ are called the boundary layer stress coefficients and we define $f^{i} \equiv 0$ if $i<0$. Introducing the transformation (18) and the expansions (6) and (19) into the exact theory (2-4) and equating coefficients of like powers of $h$, we find that the coefficients of $h^{n}$ yield

$$
\begin{array}{ccc}
f_{z x, \xi}^{n}+f_{x, \xi}^{n}=-f_{x y, y}^{n-1}, \quad f_{z, 5}^{n}+f_{z x, \xi}^{n}=-f_{z y, y}^{n-1}, \quad f_{z y, \zeta}^{n}+f_{x y, \xi}^{n}=-f_{y, y}^{n-1} ; & (20 \mathrm{a}, \mathrm{b}, \mathrm{c}) \\
\nabla^{2} f_{x}^{n}+\Gamma_{, \xi \xi}^{n}=-f_{x, y y}^{n-2}, \quad \nabla^{2} f_{y}^{n}=-f_{y, y \nu}^{n-2}-\Gamma_{, y y}^{n-2}, \quad \nabla^{2} f_{z}^{n}+\Gamma_{, \zeta \zeta}^{n}=-f_{z, y y}^{n-2} & (21 \mathrm{a}, \mathrm{b}, \mathrm{c}) \\
\nabla^{2} f_{z x}^{n}+\Gamma_{, \zeta \xi}^{n}=-f_{z x, y y}^{n-2}, \quad \nabla^{2} f_{z y}^{n}=-\Gamma_{, \zeta y}^{n-1}-f_{z y, y y}^{n-2}, & (21 \mathrm{~d}, e, f) \\
\nabla^{2} f_{x y}^{n}=-\Gamma_{, \xi y}^{n-1}-f_{x y, y y}^{n-2} ; &
\end{array}
$$

${ }^{8}$ For more detailed descriptions of these methods see [3-8]. 


$$
\begin{gathered}
f_{z x}^{n}(\xi, y, \pm 1)=f_{z}^{n}(\xi, y, \pm 1)=f_{z y}^{n}(\xi, y, \pm 1)=0 \\
f_{x}^{n}(0,0, \zeta)=L^{n}(0, \zeta), \quad f_{z x}^{n}(0,0, \zeta)=R^{n}(0, \zeta), \quad f_{x y}^{n}(0,0, \zeta)=Q^{n}(0, \zeta),
\end{gathered}
$$

where,

$$
\nabla^{2} f \equiv \frac{\partial^{2} f}{\partial \xi^{2}}+\frac{\partial^{2} f}{\partial \zeta^{2}} \quad \text { and }, \quad \Gamma^{n}(\xi, y, \zeta)=\frac{1}{1+\nu}\left(f_{x}^{n}+f_{\nu}^{n}+f_{z}^{n}\right) .
$$

Equations (20-22) are written for $y=0$, i.e. in the normal plane to $B$ at $P$.

To complete the formulation of the boundary layer problem we require that each $f$ approaches or "matches" the corresponding interior stress as $h \rightarrow 0$, or equivalently from (18) as $\xi \rightarrow-\infty$. More precisely, the matching conditions, or the asymptotic forms of the boundary layer stress coefficients are obtained $[3,4,5]$ by assuming that each interior stress coefficient, $\sigma^{i}(x, 0, \zeta)$, possesses a Taylor series expansion about $x=0$

$$
\sigma^{i}(x, 0, \zeta)=\sum_{i=0}^{\infty} A_{i}^{i}(\zeta) x^{i}
$$

where,

$$
\begin{aligned}
& A_{i}^{i}(\zeta)= \begin{cases}\frac{1}{j !} \frac{\partial^{i} \sigma^{i}(0,0, \zeta)}{\partial x^{i}}, & \text { if } \quad i \geq 0\end{cases} \\
& 0, \text { if } i<0 .
\end{aligned}
$$

It follows from (5), (18), (23) and (24a) that in the neighborhood of $x=0$,

$$
\sigma(x, 0, \zeta ; h) \sim \sum_{n=0}^{\infty} D^{n}(\xi, 0, \zeta) h^{n},
$$

where

$$
D^{n}(\xi, 0, \zeta)=\sum_{i=0}^{n} A_{i}^{n-i}(\zeta) \xi^{j}
$$

are the interior stress coefficients near $x=y=0$ as functions of $(\xi, \zeta)$. If we define the "reduced boundary layer stress coefficients" $F^{n}(\xi, 0, \zeta)$ as,

$$
F^{n}(\xi, 0, \zeta) \equiv f^{n}(\xi, 0, \zeta)-D^{n}(\xi, 0, \zeta), \quad n=0,1, \cdots,
$$

then employing (19), (25) and the definitions of $f(\xi, 0, \zeta ; h)$ and $\sigma(x, 0, \zeta ; h)$ the asymptotic form for the $f^{n}$ is obtained by identifying each $f^{n}$ with the corresponding $D^{n}$ near $x=0$ as $h \rightarrow 0$. We write this condition, using (18) and (26a), as:

$$
\lim _{\xi \rightarrow-\infty} F^{n}(\xi, 0, \zeta)=0 .
$$

In obtaining (26) we assume that the terms in $f^{n}$ which vanish as $\xi \rightarrow-\infty$ do so faster than any negative power of $\xi$.

The boundary layer problem, (20-22), (24) and (26), is systematically analyzed in the next section.

5. Analysis of the boundary layer. For each $n$, the boundary layer problem separates into two distinct systems which are called Problem $P^{n}$ and Problem $T^{n}$. Problem $P^{n}$ involves only the coefficients $f_{x}^{n}, f_{y}^{n}, f_{z}^{n}$, and $f_{z x}^{n}$ and consists of Eqs. (20a, b), (21a, b, c, d), 
the first two of (22a, b) and (24) and (26) for these coefficients. Problem $T^{n}$ is concerned with the two remaining coefficients and consists of $(20 \mathrm{c}),(21 \mathrm{e}, \mathrm{f})$, the last of $(22 \mathrm{a}, \mathrm{b})$ and (24) and (26) for these coefficients.

We shall associate with the $F^{m}$ of Problem $P^{n}$ a function $\phi^{n}(\xi, 0, \xi)$ which may be the solution of the following boundary value problem on the semi-infinite strip $D$, $|\zeta| \leq 1, \xi \leq 0$

$$
\begin{gathered}
\nabla^{4} \phi^{n}=0 ; \\
\phi_{, \xi \xi \xi}^{n}(\xi, 0, \pm 1)=k^{n}(\xi), \quad \phi_{, \xi \zeta}^{n}(\xi, 0, \pm 1)=0 ; \quad \lim _{\xi \rightarrow-\infty}\left[\phi_{, \zeta \zeta}^{n}, \phi_{, \xi \xi}^{n}\right]=0 ; \\
\phi_{, . \zeta 5}^{n}(0,0, \zeta)=\alpha^{n}(0, \zeta), \quad \phi_{, \xi \zeta}^{n}(0,0, \zeta)=\beta^{n}(0, \zeta),
\end{gathered}
$$

where $\alpha^{n}$ and $\beta^{n}$ are respectively even and odd functions of $\zeta$ and $\int_{0}^{\infty} k^{n}(\xi) d \xi=0$. It can be shown by an elementary calculation that if $\phi_{.55}^{n}, \phi_{, \xi 5}^{n}$ and $\phi_{.5}^{n}$ are uniformly continuous functions of $\zeta$ and if $\phi^{n}, \phi_{, \xi}^{n}$ and $\phi_{, \xi}^{n}$ are single valued functions, then,

$$
\int_{-1}^{1} \alpha^{n}(0, \zeta) d \zeta=0
$$

Using (11), (13) and (17) we can show that

$$
F_{x}^{0}=\phi_{.5 \zeta}^{0}, \quad F_{z}^{0}=\phi_{. \xi \xi}^{0}, \quad F_{z x}^{0}=-\phi_{.5 \xi}^{0}, \quad F_{\nu}^{0}=\nu \nabla^{2} \phi^{0}
$$

is a solution of Problem $P^{0}$ if $\phi^{0}$ is a solution of (27) with,

$$
k^{0}(\xi) \equiv 0, \quad \alpha^{0}(0, \zeta)=L^{0}(0, \zeta)-S_{x}^{0}(0,0), \quad \beta^{0}(0, \zeta)=-R^{0}(0,0) .
$$

Application of (28) to (29b) gives the first boundary condition at $y=0$ for the zeroth order interior problem as,

$$
S_{x}^{0}(0,0)=\frac{1}{2} \int_{-1}^{1} L^{0}(0, \zeta) d \zeta .
$$

Similarly, by employing (11), (13) and (17) Problem $T^{0}$ has the solution,

$$
F_{z y}^{0}=\psi_{.5}^{0}, \quad F_{x y}^{0}=\psi_{. \xi}^{0}
$$

if $\psi^{0}$ is a solution of the following boundary value problem on $D$

$$
\begin{aligned}
\nabla^{2} \psi^{0} & =0 ; \\
\psi_{. \zeta}^{0}(\xi, 0, \pm 1) & =0 ; \quad \lim _{\xi \rightarrow-\infty}\left\{\psi_{. \xi}^{0}, \psi_{. \zeta}^{0}\right\}=0 ; \\
\psi_{. \xi}^{0}(0,0, \zeta) & =Q^{0}(0, \zeta)-S_{x y}^{0}(0,0) .
\end{aligned}
$$

Existence of a solution of (31b) requires that the integral around the boundary of the normal derivative of $\psi^{0}$ vanishes. This yields the second and final boundary condition for the zeroth order interior problem at $y=0$ as,

$$
S_{x y}^{0}(0,0)=\frac{1}{2} \int_{-1}^{1} Q^{0}(0, \zeta) d \zeta .
$$

The solution to Problem $P^{1}$ can be reduced to solving (27) with

$$
\begin{gathered}
k^{1}(\xi)=\psi_{, \nu}^{0}(\xi, 0,+1), \quad \alpha^{1}(0, \zeta)=L^{1}(0, \zeta)-S_{x}^{1}(0,0)+\psi_{, \nu}^{0}(0,0, \zeta), \\
\beta^{1}(0, \zeta)=-R^{1}(0, \zeta)
\end{gathered}
$$


and

$$
\begin{gathered}
F_{x}^{1}=\phi_{.55}^{1}-\psi_{, \nu}^{0}, \quad F_{z}^{1}=\phi_{, x x}^{1}-\psi_{. \nu}^{0}, \quad F_{z x}^{1}=-\phi_{.5 \xi}^{1}, \\
F_{y}^{1}=\nu \nabla^{2} \phi^{1}+2 \psi_{, y}^{0} .
\end{gathered}
$$

From (28) and the second of (32a) we find the first boundary condition at $y=0$ for the first order interior problem as,

$$
S_{x}^{1}(0,0)=\frac{1}{2} \int_{-1}^{1}\left[L^{1}(0, \zeta)+\psi_{. y}^{0}(0,0, \zeta)\right] d \zeta .
$$

If we set

$$
F_{z \nu}^{1}=\Xi_{, \xi}^{1}-\nu \phi_{, 5 \nu}^{0}, \quad F_{x \nu}^{1}=\Xi_{, 5}^{1}-\nu \phi_{, \xi \nu}^{0},
$$

then (34a) is a solution of Problem $T^{1}$ if $\Xi^{1}(\xi, 0, \zeta)$ is a solution of the following boundary value problem on $D$ :

$$
\begin{aligned}
\nabla^{2} \Xi^{1} & =(1-\nu) \int_{\xi}^{\infty} \nabla^{2} \phi_{, \zeta \nu}^{0} d \xi \\
\Xi_{, \xi}^{1}(\xi, 0, \pm 1) & =0, \quad \lim _{\xi \rightarrow-\infty}\left\{\Xi_{, \xi}^{1}, \Xi_{, \zeta}^{1}\right\}=0 \\
\Xi_{, \zeta}^{1}(0,0, \zeta) & =-Q^{1}(0, \zeta)+S_{x y}^{1}(0,0)+\nu \int_{-1}^{\zeta} R_{, \nu}^{0}(0, \zeta) d \zeta .
\end{aligned}
$$

It can be shown by an elementary calculation that if $\Xi_{, 5}^{1}$ is a uniformly continuous function of $\zeta$ and if $\Xi$ is a single valued function then,

$$
S_{x y}^{1}(0,0)=\frac{1}{2} \int_{-1}^{1}\left[Q^{1}(0, \zeta)+\nu \zeta R_{, \nu}^{0}(0, \zeta)\right] d \zeta .
$$

This is the second and final boundary condition for the first order interior problem. Higher order boundary layer approximations may be obtained by proceeding in a similar manner.

From Problems $P^{n}$ and $T^{n}$ given above we observe that, in general, $F_{z}, F_{z x}$ and $F_{z y}$ do not vanish, while in the Appendix it is shown that the corresponding interior stresses, $\sigma_{z}, \sigma_{z x}$ and $\sigma_{z y}$ do vanish. Thus there is a contribution to the transverse normal and shear stresses only in the boundary layer and we would therefore expect the plane stress theory, i.e. the zeroth order interior problem, to yield a "good" approximation to the exact solution in the interior. This has been verified for the problem of an infinite plate with a circular hole stretched by a uniaxial force at infinity. The three-dimensional corrections to the plane stress theory yield only "small" changes in the stress concentration factor. These results will be reported in detail elsewhere.

6. Summary. In the preceding sections we have shown, using systematic expansion procedures, the relation of the plane stress theory to the exact theory. The plane stress theory appears as the zeroth order interior problem, (11), (13) and (17) with $n=0$ and (30), the solutions of which supply a first approximation to the three-dimensional stress distribution. If we introduce an Airy stress function, $G^{0}(x, y)$, such that,

$$
S_{x}^{0}=G_{, y y}^{0}, \quad S_{y}^{0}=G_{, x x}^{0}, \quad S_{x y}^{0}=-G_{, x y}^{0},
$$

then the zeroth order interior problem can be written in the familiar form 


$$
\Delta^{2} G^{0}=0
$$

and

$$
S_{x}^{0}=G_{, y y}^{0}=\frac{1}{2} \int_{-1}^{1} L^{0} d \zeta, \quad S_{x y}^{0}=-G_{, x y}^{0}=\frac{1}{2} \int_{-1}^{1} Q^{0} d \zeta, \quad \text { on } B .
$$

In addition, the expansion procedure provides "three-dimensional corrections" to the plane stress theory. The first correction is in the boundary layer and is obtained from the solutions of $P^{0}$ and $T^{0}$. The next correction is obtained from the solutions of the first order interior problem, (11), (13) and (17) with $n=1$ and (33), and Problems $P^{1}$ and $T^{1}$. We notice from (32) and (34) that the solutions of the zeroth order problems provide inhomogeneous terms for the first order problems. We define an $N$ th approximation to the exact theory by introducing $\sigma^{(N)}(x, y, \zeta ; h)$ as the generic symbol for the stress components of this approximation where,

$$
\sigma^{(N)}(x, y, \zeta ; h)=\sum_{n=0}^{N}\left[\sigma^{n}(x, y, \zeta)+F^{m}(x / h, y, \zeta)\right] h^{n}
$$

Here $\sigma^{n}(x, y, \zeta)$ and $F^{n}(x / h, y, \zeta)$ are, respectively, the interior and reduced boundary layer stress coefficients of order $n$ obtained from the solutions of the $n$th order interior and boundary layer problems respectively.

\section{References}

1. S. Timoshenko and J. Goodier, Theory of elasticity, 2nd ed., McGraw-Hill, New York, 1951

2. G. Kïrsch, Die Theorie der Elastizität und die Bedürfnisse der Festigkeitslehre, Z. ver. Deut. Ing. 42, 797 (1898)

3. E. L. Reiss, Symmetric bending of thick circular plates, to appear

4. E. L. Reiss, $A$ theory for the small rotationally symmetric deformations of cylindrical shells, Communs. on Pure and Appl. Math. 13, 531-550 (1960)

5. E. L. Reiss, $A$ theory for the small unsymmetric deformations of cylindrical shells, Rep. IMM-NYU 274, Institute of Mathematical Sciences, New York Univ., 1960

6. K. O. Friedrichs, The edge effect in the bending of plates, Reissner Anniv. Vol., 197-210, Edwards, Ann Arbor, Mich., 1949; Kirchoff's boundary conditions and the edge effect for elastic plates, Proc. Sym. in Appl. Math. 3, McGraw-Hill, New York, 1950, pp. 117-124

7. K. O. Friedrichs and R. F. Dressler, $A$ boundary layer theory for elastic bending of plates, Communs. on Pure and Appl. Math., 14 (1961)

8. K. O. Friedrichs, Asymptotic phenomena in mathematical physics, Bull. Am. Math. Soc. 61, 485-504 (1955)

9. E. Reissner, On the calculation of three-dimensional corrections for the two-dimensional theory of plane stress, Proc. $15^{\text {th }}$ Bull. Eastern Photoelasticity Conf., 23-31, Boston, 1942

\section{Appendix}

Recalling that $\sigma_{z}^{n}$ and $\Omega^{n}$ are even functions of $\zeta$ and that $\sigma_{z x}^{n}$ and $\sigma_{z y}^{n}$ are odd functions of $\zeta$, we prove the Theorem. $\sigma_{z x}^{n}=\sigma_{z y}^{n}=\sigma_{z}^{n}=0$ and $\Omega^{n}=\Omega^{n}(x, y)$ for all $n$.

The proof is by induction. Let $\sigma_{z x}^{n}=\sigma_{z y}^{n}=\sigma_{z}^{n}=\sigma_{z x}^{n+1}=\sigma_{z y}^{n+1}=0$, and $\Omega^{n}=\Omega^{n}(x, y)$ and $\Omega^{n+1}=\Omega^{n+1}(x, y)$. Then from (7c) and (9) we immediately conclude that $\sigma_{z}^{n+1}=\sigma_{z}^{n+2}=0$ and hence from (8c), $\Omega^{n+2}=\Omega^{n+2}(x, y)$. It follows from (8d, e) and (9) and the inductive assumption that, $\sigma_{z x}^{n+2}=\sigma_{z y}^{n+2}=0$. The induction is completed by reference to Eqs. (10-12). Thus,

$$
\sigma_{z x} \sim \sum_{i=0}^{\infty} \sigma_{z x}^{i} h^{i}=0, \quad \sigma_{z y} \sim \sum_{i=0}^{\infty} \sigma_{z y}^{i} h^{i}=0, \quad \sigma_{z} \sim \sum_{i=0}^{\infty} \sigma_{z}^{i} h^{i}=0 .
$$

